BRE 22106

\title{
Action spectrum of the retinal mechanism mediating nocturnal light-induced suppression of rat pineal gland N-acetyltransferase
}

\author{
David M. Bronstein ${ }^{2}$, Gerald H. Jacobs ${ }^{1}$, Katherine A. Haak ${ }^{1}$, \\ Jay Neitz ${ }^{1}$ and Loy D. Lytle ${ }^{1}$

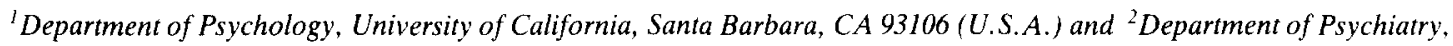 \\ Mental Health Research Institute, The University of Michigan, Ann Arbor, MI 48109 (U.S.A.)
}

(Accepted 5 November 1986)

Key words: Pineal gland; N-Acetyltransferase activity; Rod photopigment; Albino rat

\begin{abstract}
The spectral properties of the retinal mechanism mediating the inhibitory effects of nocturnal light on pineal gland $\mathrm{N}$-acetyltransferase (NAT) activity were determined. Pineal gland NAT activity declined linearly in albino rats exposed to different irradiances of a 460 or $580 \mathrm{~nm}$ monochromatic light during the middle of the dark phase of the cycle. The difference in sensitivity to the test lights is that predicted for a photopigment having peak absorbance at $495 \mathrm{~nm}$, suggesting the inhibition of pineal gland $\mathrm{N}$-acetyltransferase activity is mediated by the photopigment found in rat rods.
\end{abstract}

Acetyl-CoA arylamine $N$-acetyltransferase $(N$ acetyltransferase; NAT; EC 2.3.1.5) plays an important role in the synthesis of the pineal gland methoxyindoleamine hormone, melatonin, by catalyzing the conversion of the substrate, serotonin, to the intermediate product, $\mathrm{N}$-acetylserotonin ${ }^{12,18}, \mathrm{~N}$ Acetylserotonin then undergoes catalysis by the enzyme hydroxyindole-O-methyltransferase (HIOMT; EC 2.1.1.4) to form melatonin ${ }^{12,18}$. Changes in environmental lighting influence the pineal gland in most mammals via a neural circuit that links the retina to the pineal gland ${ }^{13,17,19}$. Pineal gland NAT and HIOMT activities as well as $N$-acetylserotonin and melatonin concentrations are highest during darkness and lowest during the light phase of the day:night cycle. The normal nocturnal elevations in NAT activity and melatonin synthesis decline rapidly in animals exposed briefly to light, even when the presentations of light last for only 1 min or less ${ }^{10,11,21}$.

Although light-induced suppression of pineal gland NAT activity has been extensively described ${ }^{2}$. 10.11 .15 .21 , relatively less is known about the spectral properties of light that make it an effective stimulus. In one experiment, greenish light $(510-550 \mathrm{~nm})$ appeared to be a more effective suppressor of rat pineal gland HIOMT activity than lights of longer or shorter wavelengths $s^{4,5}$. Light drawn from the $435-500 \mathrm{~nm}$ part of the spectrum has also been shown to be effective in causing reductions in pineal gland melatonin levels in Syrian hamsters ${ }^{3}$. A natural interpretation is that the retinal mechanisms initiating this response have maximum absorption in the blue-to-green part of the spectrum.

This study was conducted to specify more precisely these retinal mechanisms in the rat. Our approach has two unique features. First, action spectra were determined for nocturnal light-induced inhibition of pineal gland NAT activity. Earlier studies only reported response measures; these cannot unambiguously specify the spectral absorption properties of the mechanisms involved. Second, a recent investigation $^{16}$ of rat vision involving both electrophysiological and behavioral measures reveals that the rat retina contains only two types of photopigment, one type

Correspondence: G.H. Jacobs, Department of Psychology, University of California, Santa Barbara, CA 93106, U.S.A. 
normally found in rods having peak absorption $\left(\lambda_{\max }\right)$ at about $498 \mathrm{~nm}$, and a second type, apparently contained in cones, having a $\lambda_{\text {max }}$ at about $510 \mathrm{~nm}$. Prior knowledge of the identity of these two mechanisms allowed us to assess the effects of only two monochromatic lights on the pineal gland NAT response and establish which of these two mechanisms might be involved in mediating the inhibitory effect.

Adult ( 90 days old) male and female SpragueDawley albino rats were bred and reared in our vivarium from a parent stock of animals purchased commercially (Charles River Labs., Wilmington, MA). All animals were offered ad libitum access to food and tap water, maintained under $22-23{ }^{\circ} \mathrm{C}$ ambient temperature conditions at $55 \%$ relative humidity, and exposed to a 12:12 h light:dark cycle (lights on at $07.00 \mathrm{~h}$ ). Colony illumination was provided by Westinghouse Inside Frosted $150 \mathrm{~W}$ A-23 incandescent bulbs. These lights yielded an average illuminance of 32 lux (range 16-48 lux) inside the colony cages.

An enclosure with two light proof compartments was used to expose animals to nocturnal light. The upper compartment $(33 \times 33 \times 33 \mathrm{~cm})$ contained a $100 \mathrm{~W}$ tungsten-halogen lamp (Halo Star, Osram) driven by a $24 \mathrm{~V} \mathrm{DC}$ power supply underrun at $22 \mathrm{~V}$. Monochromatic light of $460 \mathrm{~nm}$ or $580 \mathrm{~nm}$ with irradiances varying over a $3 \log _{10}$ range was produced by placing interference filters (Ditric, half-energy passband $=10 \mathrm{~nm}$ ) and Wratten neutral density filters (Eastman Kodak, Rochester, NY) between the light source and two aspherical condensing lenses that focussed the light beam onto the center of a $2.4 \mathrm{~cm}$ dia electronically driven shutter (Ralph Gerbrands, Arlington, MA). An open top cylinder (18 $\mathrm{cm}$ diameter $\times 29 \mathrm{~cm}$ high), made of highly diffusing white plastic and located such that its top was $1 \mathrm{~cm}$ below the shutter, was used to confine the movements of the animals in the lower compartment.

Animals were placed individually into the test chamber in darkness during the middle of the night and exposed for $60 \mathrm{~s}$ to one of 5 different irradiances. The irradiances for each of the monochromatic test lights were measured with a silicon diode photodetector (PIN 10 DL, United Detector Technology) placed at the approximate position of the rat's head in the exposure chamber. Following the 60 $s$ exposure, animals remained in darkness until they were killed $30 \mathrm{~min}$ later. Control animals were placed in the test chamber for $60 \mathrm{~s}$ but were not exposed to light. They were also returned to their cages and kept in darkness until they were killed 30 min later.

Animals were killed by cervical decapitation under dim red light (longer than $680 \mathrm{~nm}$ ) shown previously to not affect nocturnal pineal gland NAT activity ${ }^{20}$. Pineal glands were removed within $30 \mathrm{~s}$ and frozen at $-70{ }^{\circ} \mathrm{C}$, and then assayed for NAT activity using a modification ${ }^{1}$ of the method of Deguchi and Axel$\operatorname{rod}^{8}$. Whole pineal gland NAT activity was calculated as the nmol of tryptamine converted to $\mathrm{N}$-acetyltryptamine per pineal gland per hour.

Nocturnal pineal gland NAT activity in control animals not exposed to light was typically high (approximately $20 \mathrm{nmol}$ of $N$-acetyltryptamine formed per pineal gland per hour). One min exposure to 12 or 13 $\log _{10}$ quanta $\cdot \mathrm{cm}^{-2} \cdot \mathrm{s}^{-1}$ of the $460 \mathrm{~nm}$ or $580 \mathrm{~nm}$ test lights suppressed pineal gland NAT activity to $8 \%$ or $5 \%$, respectively, of the dark control values (Fig. 1, insert). Pineal gland enzyme activity was reduced linearly in animals exposed to the $460 \mathrm{~nm}$ test light over the range of $10-11 \log _{10}$ light quanta $\cdot \mathrm{cm}^{-2} \cdot \mathrm{s}^{-1}$; in contrast, the linear range for suppression of pineal gland NAT activity caused by the $560 \mathrm{~nm}$ light stimulus occurred over an irradiance range of 11-12 quan$\mathrm{ta} \cdot \mathrm{cm}^{-2} \cdot \mathrm{s}^{-1}$. Essentially complete inhibition of the enzyme was observed above irradiances of 11.5 and

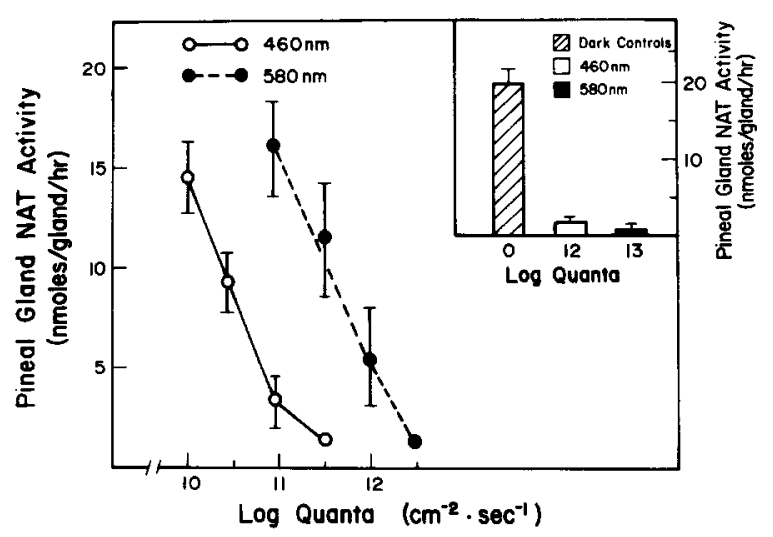

Fig. 1. Effects of different irradiances of two monochromatic lights on nocturnal pineal gland NAT activity. All values are the means ( \pm S.E.M.) nmol of acetyltryptamine formed pineal gland $\cdot \mathrm{hr}^{-1}$ ( $n=$ at least 6 animals per group). Inset: pineal gland NAT activity in dark exposed control animals or in animals exposed to high irradiance levels (12.5 log quanta $\cdot \mathrm{cm}^{-2} \cdot \mathrm{s}^{-1}$ ) of the $460 \mathrm{~nm}$ and $580 \mathrm{~nm}$ monochromatic test light stimuli $\left(F_{2,23}=61.7 ; P<0.001\right.$ compared to dark exposed control values). 
$12.5 \log$ quanta for the $460 \mathrm{~nm}$ and $580 \mathrm{~nm}$ test stimuli, respectively (Fig. 1). Regression lines of best fit were calculated for the data obtained for each of the test lights over irradiances that caused linear inhibition of enzyme activity. The regression lines for the two test stimuli had similar slopes, and the average sensitivity difference between these monochromatic lights was calculated to be $1.02 \log _{10}$ units. An iterative computer routine was used to determine the spectral peak of a photopigment that would yield this sensitivity difference. This routine compared the sensitivity differences between the two test lights to each of a series of photopigment spectra whose $\lambda_{\max }$ values cover the range from $470 \mathrm{~nm}$ to $530 \mathrm{~nm}$ in steps of 1 $\mathrm{nm}$. The procedure is predicated on the fact that, when appropriately scaled, the spectra for all visual photopigments can be accounted for by a small number of nomograms that vary only in their spectral positioning ${ }^{7.9}$. The nomogram-based curve found to provide the best fit to the biochemical data from the total array of all such curves had a $\lambda_{\max }$ value of 495 nm (Fig. 2, right panel).

Our results are consistent with the hypothesis that nocturnal light-induced inhibition of pineal gland NAT activity is most likely mediated by rod photopigments in the retina of the rat. This conclusion is based on the argument illustrated in Fig. 2. Shown in the left and center panels are spectral sensitivity curves for the only two photopigments clearly identi- fied in the retina of the rat ${ }^{16}$. As noted previously, one of these photopigments is found in rods, and has a spectral sensitivity curve with a $\lambda_{\max }=498 \mathrm{~nm}$; the second pigment appears to be associated with cones, and has a spectral sensitivity curve with a $\lambda_{\max }$ at 510 $\mathrm{nm}^{16}$. To determine if either one or both of these photopigments was responsible for the inhibition of NAT activity, animals were exposed to different irradiance levels of two test lights having wavelengths of 460 and $580 \mathrm{~nm}$. These two wavelengths were selected because each would be expected to robustly stimulate the two pigments, yet their predicted effects on the two pigments would be quite different. The difference in sensitivity of pineal gland NAT to the 460 and $580 \mathrm{~nm}$ lights (vertical lines in the left and center panels in Fig. 2) would be predicted to $0.89 \log _{10}$ units if the $498 \mathrm{~nm}$ pigment was activated whereas the sensitivity difference would be only $0.47 \log _{10}$ units if the $510 \mathrm{~nm}$ pigment was the transducer. In behavioral tests where both of the retinal pigments are operative their outputs are simply summed ${ }^{16}$. In such cases the difference in sensitivity to the two test lights would lie somewhere between 0.47 and $0.89 \log _{10}$ units. Note that although the two pigment curves in the left and center panels of Fig. 2 have been arbitrarily drawn to the same peak sensitivity, that fact is not critical for the argument. The predicted differences in sensitivity of these two pigments to the two test wavelengths are independent of their absolute sensitivities. For

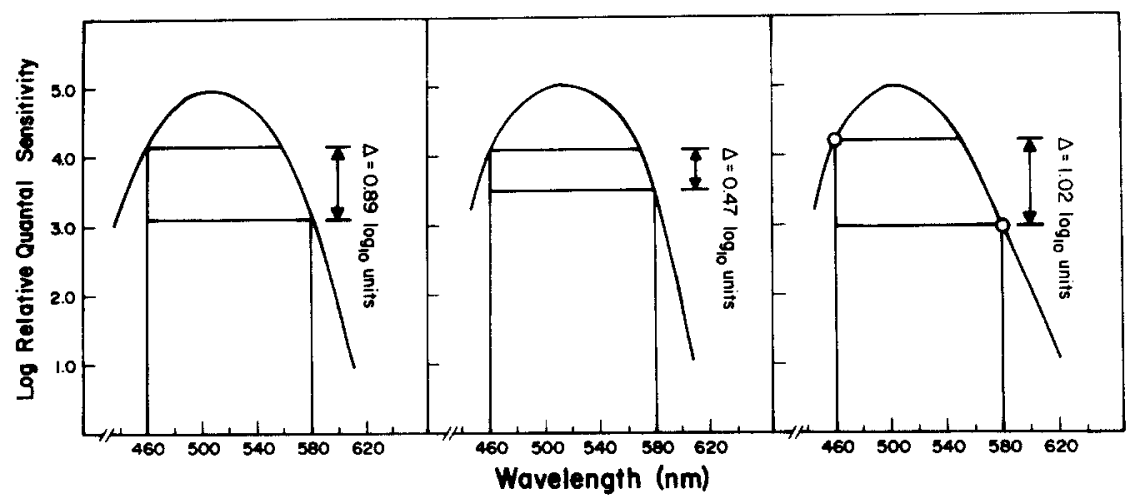

Fig. 2. Spectral sensitivity curves for the two spectral mechanisms (left and middle panels) in the rat retina ${ }^{16}$. The right panel shows a computer-derived photopigment nomogram that best fits the data (open circles) describing the inhibitory effects of nocturnal monochromatic light on pineal gland NAT activity. The vertical lines drawn at 460 and $580 \mathrm{~nm}$ in each panel represent the wavelengths of the two monochromatic lights used to suppress pineal gland NAT activity. The relative differences in quantal sensitivity were predicted to be $0.89 \log _{10}$ units or $0.47 \log _{10}$ units if only rod $\left(\lambda_{\max }=498 \mathrm{~nm}\right)$ or cone $\left(\lambda_{\max }=510 \mathrm{~nm}\right)$ photopigments, respectively, mediated the inhibitory effects of the $460 \mathrm{~nm}$ and $580 \mathrm{~nm}$ test lights on pineal gland NAT activity. The theoretical curve that best fits the experimental results is that for a photopigment with a $\lambda_{\max }$ of $495 \mathrm{~nm}$. 
comparison purposes the panel on the right in Fig. 2 shows the spectral sensitivity for the nomogrambased curve derived by the computer analysis that best fits the data obtained in our experiments. As noted, the actual mean difference in sensitivity between the two test lights over the linear range of inhibition of pineal gland NAT activity was $1.02 \log _{10}$ units (Fig. 2, right panel). The spectral sensitivity of the mechanism mediating nocturnal light-induced inhibition of the pineal gland enzyme response is thus close to that predicted based on the operation of a rat rod photopigment (an obtained peak of $495 \mathrm{~nm}$ vs a prediction of $498 \mathrm{~nm}$ ).

It has been suggested previously ${ }^{3,19}$ that both rods and short wavelength cones might mediate nocturnal light-induced suppression of rodent pineal gland melatonin. This interpretation was based on earlier evidence suggesting that the rat retina might contain 3 cone pigments, including one with peak sensitivity in the short wavelengths ${ }^{6}$. However, since it now appears that the rat retina does not contain such a short wavelength-sensitive photopigment ${ }^{16}$, this possibility can be ruled out. Although a pigment with a $\lambda_{\max }$ at $510 \mathrm{~nm}$ has been identified in the rat retina ${ }^{16}$, under the present test conditions the suppression of pineal gland NAT activity clearly results from the operation of a mechanism having a $\lambda_{\max }$ of $495 \mathrm{~nm}$. That figure is so close to the one predicted by the rod photopigment that the conclusion that rod photopigments alone mediate the nocturnal light-induced suppression of pineal gland biochemistry seems most likely. This idea is compatible with the recent demonstration ${ }^{20}$ that the mechanism responsible for the entrainment of the hamster activity cycle also appears to have the spectral sensitivity of a rod photopigment.

The activity of the $510 \mathrm{~nm}$ mechanism can be easily demonstrated electrophysiologically in recordings from the retina of the rat, and its contribution to various behavioral responses has been documented ${ }^{16}$. It

1 Altar, A., Motroni, T.P. and Lytle, L.D., Functional synaptogenesis and the rat pineal gland: a pharmacological investigation, J. Neural Transm., 58 (1983) 231-241.

2 Brainard, G.C., Richardson, B.A., King, T.S., Matthews, S.A. and Reiter, R.J., The suppression of pineal melatonin content and $\mathrm{N}$-acetyltransferase activity by different light irradiances in the Syrian hamster: a dose-response relationship, Brain Research, 113 (1983) 293-296.

3 Brainard, G.C., Richardson, B.A., King, T.S. and Reiter, may seem surprising that this mechanism, presumably a cone photopigment, makes no contribution to the light-induced suppression of nocturnal pineal gland NAT activity. Since cone vision is found routinely to have a higher threshold than rod vision, one possible explanation is that the light levels employed in this experiment were below cone thresholds. However, a comparison of the luminance levels required to yield rat cone-based vision in behavioral experiments ${ }^{16}$ to the levels used in the present experiments suggests that stimuli at the upper ends of the irradiance ranges used here probably exceeded the behaviorally measured cone threshold. For instance, measurements made from a magnesium block placed in the test chamber at the approximate position of the rat's eyes indicated that the $580 \mathrm{~nm}$ light that decreased NAT activity to $25 \%$ of dark values had a luminance of $0.6 \mathrm{~cd} \cdot \mathrm{m}^{-2}$. Light of that intensity is sufficient to yield behavior based on the operation of both rods and cones, but it is not intense enough to ensure that only cones are operative ${ }^{16}$. Unfortunately, intensity increases above those levels cause maximal suppression of pineal gland NAT activity, thus making it impossible to know whether still higher light levels might provide evidence for cone suppression of the activity of the pineal gland enzyme. One can only conclude that if the outputs from rat cones inhibit pineal gland NAT activity they do so only at light levels higher than those required to completely suppress NAT activity and to allow cones to influence visual behavior.

This research was supported in part by grants from the National Eye Institute (EY-00105) to G.H.J., the National Institute of Mental Health (MH-31134) to L.D.L., and by a National Science and Engineering Research Council (NSERC) Predoctoral Fellowship to D.M.B.

R.J., The influence of different light spectra on the suppression of pineal melatonin content in the Syrian hamster, Brain Research, 294 (1984) 333-339.

4 Cardinali, D.P., Larin, F. and Wurtman, R.J., Action spectra for effects of light on hydroxyindole-O-methyltransferases in rat pineal, retina and Harderian gland, Endocrinology, 91 (1972) 877-886.

5 Cardinali, D.P., Larin, F. and Wurtman, R.J., Control of the rat pineal gland by light spectra, Proc. Natl. Acad. Sci. 
U.S.A., 69 (1972) 2003-2005.

6 Cicerone, C.M., Cones survive rods in the light damaged eye of the albino rat, Science, 194 (1976) 1183-1185.

7 Dawis, S.M., Polynomial expressions of pigment nomograms, Vision Res., 21 (1981) 1427-1430.

8 Deguchi, T. and Axelrod, J., Sensitive assay for serotonin $\mathrm{N}$-acetyltransferase activity in rat pineal, Anal. Biochem., 50 (1972) 174-179.

9 Ebrey, T.G. and Honig, B., New wavelength dependent visual pigment nomograms, Vision Res., 17 (1977) 147-151.

10 Illnerova, $\mathbf{H}$. and Vanecek, J., Effect of one-minute exposure to light at night on rat pineal serotonin $\mathrm{N}$-acetyltransferase, Prog. Brain Res., 52 (1979) 241-243.

11 Illnerova, H., Vanecek, J., Krecek, J., Wetterberg, L. and Saaf. J.. Effect of one minute exposure to light at night on rat pineal serotonin $\mathrm{N}$-acetyltransferase and melatonin, $J$. Neurochem., 32 (1979) 673-675.

12 Klein, D.C. Circadian rhythms in the pineal gland. In D.T. Krieger (Ed.), Endocrine Rhythms, Raven, New York, 1979, pp. 203-220.

13 Klein, D.C. and Moore, R.Y., Pineal N-acetyltransferase and hydroxyindole-O-methyltransferase: control by the retino-hypothalamic tract and the suprachiasmatic nucleus, Brain Research, 174 (1979) 245-262.

14 Klein, D.C., Moot, R., Weller, J.L., Higa, S., Markey, S.P., Creed, G.J. and Jacobowitz, D.M., Lesions of the paraventricular nucleus area of the hypothalamus disrupt the suprachiasmatic-spinal cord circuit in the melatonin rhythm generating system, Brain Res. Bull., 10 (1983) $647-652$.

15 Minneman, K.P., Lynch, H.J. and Wurtman, R.J., Relationship between environmental light intensity and retinamediated suppression of rat pineal serotonin-N-acetyltransferase, Life Sci., 15 (1974) 1791-1796.

16 Neitz, J. and Jacobs, G.H., Reexamination of spectral mechanisms in the rat, J. Comp. Psychol., 100 (1986) 21-29.

17 Reiter, R.J., The role of light and age in determining melatonin production in the pineal gland. In J. Axelrod, F. Fraschini and G.P. Velo (Eds.), The Pineal Gland and Its Endocrine Role, Raven, New York, 1983, pp. 227-241.

18 Reiter, R.J., The mammalian pineal gland: structure and function, Am. J. Anat., 162 (1981) 287-313.

19 Reiter, R.J., Action spectra, dose-response relationships, and temporal aspects of light's effects on the pineal gland, Ann. N.Y. Acad. Sci., 453 (1985) 215-231.

20 Takahashi, J.S., DeCoursey, P.J., Bauman, L. and Menaker, M., Spectral sensitivity of a novel photoreceptive system mediating entrainment of mammalian circadian rhythms, Nature (London), 308 (1984) 186-188.

21 Vanecek, J. and Illnerova, H., Night pineal N-acetyltransferase activity in rats exposed to white or red light pulses of various intensity and duration, Experientia, 38 (1982) $1318-1320$. 